Advances in Applied Physics, Vol. 1, 2013, no. 4, 143 - 147

HIKARI Ltd, www.m-hikari.com

\title{
The Light Curve in Supernova Modeled by a Continuous Radioactive Decay of ${ }^{56} \mathrm{Ni}$
}

\author{
L. Zaninetti \\ Dipartimento di Fisica \\ Università degli Studi di Torino \\ via P. Giuria 1, 10125 Torino, Italy
}

Copyright (c) 2013 L. Zaninetti. This is an open access article distributed under the Creative Commons Attribution License, which permits unrestricted use, distribution, and reproduction in any medium, provided the original work is properly cited.

\begin{abstract}
The UVOIR bolometric light curves are usually modeled by the radioactive decay. In order to model more precisely the absolute/apparent magnitude versus time relationship the continuous production of radioactive isotopes is introduced. A differential equation of the first order with separable variables is solved.
\end{abstract}

Keywords: Supernovae,evolution, nuclear physics aspects of, explosive burning in shock fronts

\section{Introduction}

The production of ${ }^{56} \mathrm{Ni}$, see [1], in the last phase of the stellar evolution has been predicted by $[2,3,4]$. After this theoretical prediction the radioactive decay was used as an explanation for the observations of the light curve of supernova $(\mathrm{SN})$, see among others $[5,6,7,8,9,10]$. At the same time the decay of ${ }^{56} \mathrm{Ni}$ produces a straight line in the absolute/apparent magnitude versus time relationship of the light curve which does not corresponds to the observations. We briefly recall that such a relationship presents a concavity. In order to explain this discrepancy between theory of decay and astronomical light curve we have developed a simple model for the continuous ${ }^{56} \mathrm{Ni}$ production. In this paper Section 2 derives and solves the differential equation which models the continuous production of ${ }^{56} \mathrm{Ni}$ and Section 3 shows the application of this new model to the light curve of two SNs. 


\section{The continuous production of radioactive iso- tope}

The decay of a radioactive isotope is modeled by the following equation

$$
-d N=\frac{N}{\tau}
$$

where $\tau$ is a constant and the negative sign indicates that $d N$ is a reduction in the number of nuclei, see [11]. The integration of this differential equation of the first order in which the variables can be separated gives :

$$
N(t)=N_{0} e^{-\frac{t}{\tau}}
$$

where $N_{0}$ is the number of nuclei at $t=0$. The half life is $T_{1 / 2}=\ln (2) \tau$. The absolute magnitude version of the previous formula is

$$
M=-C \log _{10}(N(t))=-\frac{t}{\tau}+k
$$

where $M$ is the absolute luminosity, $C$ and $k$ are two constants. This means that we are waiting for a straight line for the absolute magnitude versus time relationship. The continuous production of radioactive nuclei is modeled by the following equation

$$
-d N=\frac{N}{\tau} d t-P N^{\alpha} d t
$$

where $P$, the production, and $\alpha$, the exponent, are two adjustable parameters. In this differential equation of the first order the variables can be separated and the solution is

$$
N(t)=\frac{1}{\left(P \tau+\mathrm{e}^{\frac{(\alpha-1) t}{\tau}}\left(N_{0}^{-\alpha+1}-P \tau\right)\right)^{\frac{1}{\alpha-1}}}
$$

where the initial condition $N(0)=N_{0}$ has been used. The absolute magnitude version of the previous formula is

$$
\begin{array}{r}
M=-C \log _{10}(N(t))= \\
\frac{-\ln \left(\left(P \tau+\mathrm{e}^{\frac{(\alpha-1) t}{\tau}} N_{0}{ }^{-\alpha+1}-\mathrm{e}^{\frac{(\alpha-1) t}{\tau}} P \tau\right)^{-(\alpha-1)^{-1}}\right)+k \ln (2)+k \ln (5)}{\ln (2)+\ln (5)},
\end{array}
$$

where $M$ is the absolute magnitude and $C$ and $\alpha$ two constants. 


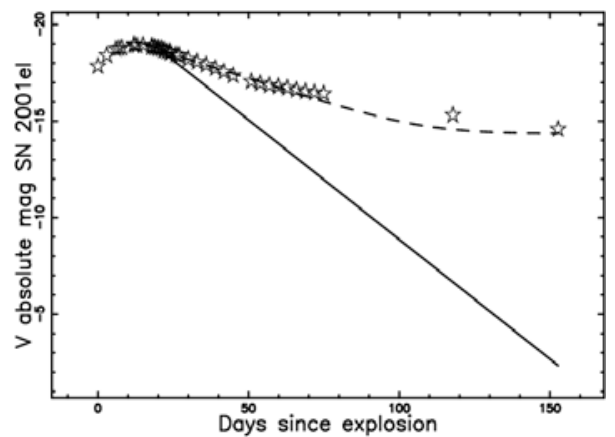

Figure 1: The $V$ light curve of SN 2001el (empty stars) in absolute magnitude, the theoretical curve as given by equation (3) when the radioactive decay of the isotope ${ }^{56} \mathrm{Ni}\left(\tau=8.757 \mathrm{~d}\right.$ or $\left.T_{1 / 2}=6.07 \mathrm{~d}, k=-18.65\right)$ was considered (full line), and the theoretical curve of the continuous production of the isotope ${ }^{56} \mathrm{Ni}\left(\tau=8.757 \mathrm{~d}\right.$ or $\left.T_{1 / 2}=6.07 \mathrm{~d}, k=-18.65, P=10^{-4}, \alpha=0.29\right)$ (dashed line).

\section{Astrophysical applications}

We plot the decay of the light curve of SN 2001el , which is of type Ia, adopting a distance modulus of $31.65 \mathrm{mag}$, see [12], the nuclear decay which according to equation (3) is a straight line, and the theoretical curve of the continuous production of radioactivity as represented by equation 6, see Figure 1. Another example is represented by SN 2001ay , the so called "the most slowly declining type Ia supernova", which has distance modulus of 35.55 mag and is of type Ia, see [8]. Figure 2 reports the light curve, the nuclear decay of the isotope ${ }^{56} \mathrm{Ni}$ and the continuous production of the isotope ${ }^{56} \mathrm{Ni}$.

\section{Conclusions}

In conclusion the continuous production of ${ }^{56} \mathrm{Ni}$ during the evolution of a $\mathrm{SN}$ is here modeled introducing two parameters $\alpha$ and $P$, see eqn.(4). The solution of this differential equation of the first order with variables which can be separated has been derived, see eqn.(5). The application of this new solution to SN 2001el and SN 2001ay produces an acceptable agreement between theory and observations over the considered temporal interval of $\approx 60 \mathrm{~d}$, see Figs. 1 and 2 . 


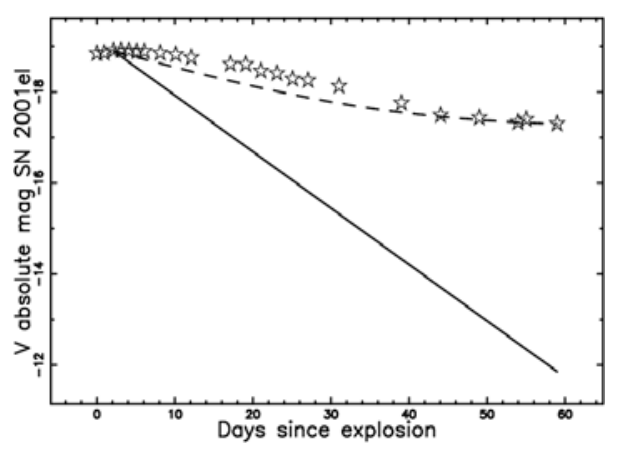

Figure 2: The $V$ light curve of SN 2001ay (empty stars) in absolute magnitude, the theoretical curve as given by equation (3) when the radioactive decay of the isotope ${ }^{56} \mathrm{Ni}\left(\tau=8.757 \mathrm{~d}\right.$ or $\left.T_{1 / 2}=6.07 \mathrm{~d}, k=-18.90\right)$ was considered (full line), and the theoretical curve of the continuous production of the isotope ${ }^{56} \mathrm{Ni}\left(\tau=8.757 \mathrm{~d}\right.$ or $\left.T_{1 / 2}=6.07 \mathrm{~d}, k=-18.90, P=0.7210^{-2}, \alpha=0.29\right)$ (dashed line).

\section{References}

[1] A. Kankainen, V.-V. Elomaa, T. Eronen, D. Gorelov, J. Hakala, A. Jokinen, T. Kessler, V. S. Kolhinen, I. D. Moore, S. Rahaman, M. Reponen, J. Rissanen, A. Saastamoinen, C. Weber, J. Äystö, Mass measurements in the vicinity of the doubly magic waiting point Ni56, Phys. Rev. C 82 (3) (2010), 034311.

[2] J. W. Truran, W. D. Arnett, A. G. W. Cameron, Nucleosynthesis in supernova shock waves, Canadian Journal of Physics 45 (1967), 2315.

[3] D. Bodansky, D. D. Clayton, W. A. Fowler, Nuclear Quasi-Equilibrium during Silicon Burning, ApJS 16 (1968), 299.

[4] S. M. Matz, G. H. Share, A limit on the production of Ni-56 in a type I supernova, ApJ 362 (1990), 235-240.

[5] P. A. Mazzali, N. Chugai, M. Turatto, L. B. Lucy, I. J. Danziger, E. Cappellaro, M. della Valle, S. Benetti, The properties of the peculiar type IA supernova $1991 \mathrm{bg}-$ II. The amount of ${ }^{\wedge} 56 \mathrm{Ni}$ and the total ejecta mass determined from spectrum synthesis and energetics considerations, MNRAS 284 (1997), 151-171.

[6] A. Elmhamdi, N. N. Chugai, I. J. Danziger, Light curves and H $\alpha$ luminosities as indicators of ${ }^{56} \mathrm{Ni}$ mass in type IIP supernovae, $A \xi A \quad 404$ (2003), 1077-1086. 
[7] M. Stritzinger, B. Leibundgut, S. Walch, G. Contardo, Constraints on the progenitor systems of type Ia supernovae, A\&A 450 (2006), 241-251.

[8] K. Krisciunas, W. Li, T. Matheson, D. A. Howell, M. Stritzinger, G. Aldering, P. L. Berlind, M. Calkins, P. Challis, R. Chornock, A. Conley, A. V. Filippenko, M. Ganeshalingam, L. Germany, S. González, S. D. Gooding, E. Hsiao, D. Kasen, R. P. Kirshner, G. H. Howie Marion, C. Muena, P. E. Nugent, M. Phelps, M. M. Phillips, Y. Qiu, R. Quimby, K. Rines, J. M. Silverman, N. B. Suntzeff, R. C. Thomas, L. Wang, The Most Slowly Declining Type Ia Supernova 2001ay, AJ 142 (2011), 74.

[9] S. Okita, H. Umeda, T. Yoshida, Mass and spatial distribution of ${ }^{56} \mathrm{Ni}$ induced by aspherical explosion of massive CO star, in: S. Kubono, T. Hayakawa, T. Kajino, H. Miyatake, T. Motobayashi, K. Nomoto (Eds.), American Institute of Physics Conference Series, Vol. 1484 of American Institute of Physics Conference Series, 2012, 418-420.

[10] T.-W. Chen, S. J. Smartt, F. Bresolin, A. Pastorello, R.-P. Kudritzki, R. Kotak, M. McCrum, M. Fraser, S. Valenti, The Host Galaxy of the Super-luminous SN 2010gx and Limits on Explosive ${ }^{56}$ Ni Production, ApJ 763 (2013), L28.

[11] F. Yang, J. H. Hamilton, Modern atomic and nuclear physics, World Scientific, Hackensack, USA, 2010.

[12] K. Krisciunas, N. B. Suntzeff, P. Candia, J. Arenas, J. Espinoza, D. Gonzalez, S. Gonzalez, P. A. Höflich, A. U. Landolt, M. M. Phillips, S. Pizarro, Optical and Infrared Photometry of the Nearby Type Ia Supernova 2001el, AJ 125 (2003), 166-180.

\section{Received: October 1, 2013}

